\title{
Electron, muon and tau magnetic moments: a theoretical update
}

\author{
M. Passera ${ }^{\text {a* }}$ \\ a Dipartimento di Fisica, Università di Padova and INFN, Via Marzolo 8, 35131 Padova, Italy \\ Recent Standard Model predictions for the anomalous magnetic moments of the electron, muon and $\tau$ lepton \\ are reviewed and compared to the latest experimental values.
}

\section{INTRODUCTION}

The gyromagnetic factor $g$ is defined by the relation between the particle's spin $\vec{s}$ and its magnetic moment $\vec{\mu}$,

$\vec{\mu}=g \frac{e}{2 m} \vec{s}$,

where $e$ and $m$ are the charge and mass of the particle. In the Dirac theory of a charged pointlike spin-1/2 particle, $g=2$. Quantum Electrodynamics (QED) predicts deviations from Dirac's value, as the charged particle can emit and reabsorb virtual photons. These QED effects slightly increase the $g$ value. It is conventional to express the difference of $g$ from 2 in terms of the value of the so-called anomalous magnetic moment, a dimensionless quantity defined as $a=(g-2) / 2$.

The measurements of the anomalous magnetic moment of the electron and the muon recently reached the fabulous relative precision of 0.7 parts per billion (ppb) [1] and 0.5 parts per million (ppm) 23], respectively. The theoretical prediction of the electron one, $a_{e}$, is rather insensitive to strong and weak interactions, hence providing a stringent test of QED and leading to the most precise determination of the fine-structure constant $\alpha$ to date [45]. On the other hand, the muon anomalous magnetic moment $a_{\mu}$ allows to test the entire Standard Model (SM) and scrutinize viable alternatives to this theory, as each of its sectors contributes in a significant way to the total prediction. Compared with $a_{e}, a_{\mu}$ is also much better suited to unveil or constrain "New Physics" (NP) effects [6]. Indeed, for a lepton

\footnotetext{
*Work supported in part by the European Community's Marie Curie Research Training Networks under contracts MRTN-CT-2004-503369 and MRTN-CT-2006-035505.
}

$l$, their contribution to $a_{l}$ is generally expected to be proportional to $m_{l}^{2} / \Lambda^{2}$, where $m_{l}$ is the mass of the lepton and $\Lambda$ is the scale of NP, thus leading to an $\left(m_{\mu} / m_{e}\right)^{2} \sim 4 \times 10^{4}$ relative enhancement of the sensitivity of the muon versus the electron anomalous magnetic moment. This more than compensates the much higher accuracy with which the $g$ factor of the latter is known. The anomalous magnetic moment of the $\tau$ lepton, $a_{\tau}$, would suit even better; however, its direct experimental measurement is prevented by the relatively short lifetime of this lepton, at least at present. The existing limits are based on the measurements of the total and differential cross sections of the reactions $e^{+} e^{-} \rightarrow e^{+} e^{-} \tau^{+} \tau^{-}$and $e^{+} e^{-} \rightarrow Z \rightarrow \tau^{+} \tau^{-} \gamma$ at LEP energies. The most stringent limit, $-0.052<a_{\tau}^{\mathrm{EXP}}<0.013$ at $95 \%$ confidence level, was set by the DELPHI collaboration [7, and is still more than an order of magnitude worse than that required to determine $a_{\tau}$.

The SM prediction $a_{l}^{\mathrm{SM}}(l=e, \mu$ or $\tau)$ is usually split into three parts: QED, electroweak (EW) and hadronic. Here we provide a summary of the present status of these contributions, and a comparison with the current experimental values.

\section{QED EFFECTS: PREAMBLE}

The QED part of the anomalous magnetic moment $a_{l}$ of a charged lepton $l=e, \mu$ or $\tau$ arises from the subset of SM diagrams containing only leptons and photons. For each of the three leptons $l$, of mass $m_{l}$, this dimensionless quantity can be cast in the general form [8]

$a_{l}^{\mathrm{QED}}=A_{1}+A_{2}\left(\frac{m_{l}}{m_{j}}\right)+A_{2}\left(\frac{m_{l}}{m_{k}}\right)+A_{3}\left(\frac{m_{l}}{m_{j}}, \frac{m_{l}}{m_{k}}\right)$, 
where $m_{j}$ and $m_{k}$ are the masses of the other two leptons. The term $A_{1}$, arising from diagrams containing photons and leptons of only one flavor, is mass and flavor independent. In contrast, the terms $A_{2}$ and $A_{3}$ are functions of the indicated mass ratios, and are generated by graphs containing also leptons of flavors different from $l$. The contribution of a lepton $j$ to $a_{l}^{\mathrm{QED}}$ is suppressed by $\left(m_{l}^{2} / m_{j}^{2}\right)$ if $m_{j} \gg m_{l}$, while it contains a logarithmic enhancement factor $\ln \left(m_{l} / m_{j}\right)$ if $m_{j} \ll m_{l}$. The muon contribution to $a_{e}^{\mathrm{QED}}$, for example, is thus power suppressed by a factor $\left(m_{e}^{2} / m_{\mu}^{2}\right) \sim 10^{-5}$; nonetheless, this effect is much larger than the tiny experimental uncertainty on $a_{e}$ [1] (see Sec. 3). On the contrary, the QED parts of $a_{\mu, \tau}$ beyond one-loop are dominated by the mass-dependent terms.

The functions $A_{i}(i=1,2,3)$ can be expanded as power series in $\alpha / \pi$ and computed order-byorder

$A_{i}=A_{i}^{(2)}\left(\frac{\alpha}{\pi}\right)+A_{i}^{(4)}\left(\frac{\alpha}{\pi}\right)^{2}+A_{i}^{(6)}\left(\frac{\alpha}{\pi}\right)^{3}+\cdots$.

Only one diagram is involved in the evaluation of the lowest-order (first-order in $\alpha$, second-order in the electric charge) contribution-it provides the famous result by Schwinger $A_{1}^{(2)}=1 / 2[9$. The mass-dependent coefficients $A_{2}$ and $A_{3}$ are of higher order. All results discussed below were derived using the latest CODATA [10] recommended mass ratios: $m_{e} / m_{\mu}=4.83633167(13) \times 10^{-3}$, $m_{e} / m_{\tau}=2.87564(47) \times 10^{-4}, m_{\mu} / m_{e}=$ $206.7682838(54), m_{\mu} / m_{\tau}=5.94592(97) \times 10^{-2}$, $m_{\tau} / m_{e}=3477.48(57), m_{\tau} / m_{\mu}=16.8183(27)$. The value for $m_{\tau}$ adopted by CODATA in Ref. [10] $\left(m_{\tau}=1776.99(29) \mathrm{MeV}\right)$ is based on the 2002 PDG result [1]. This PDG result remains unchanged to date [12]13.

\section{ELECTRON}

\subsection{QED Contributions}

Seven diagrams contribute to the fourth-order coefficient $A_{1}^{(4)}$, one to $A_{2}^{(4)}\left(m_{e} / m_{\mu}\right)$ and one to $A_{2}^{(4)}\left(m_{e} / m_{\tau}\right)$. As there are no two-loop diagrams contributing to $a_{e}^{\text {QED }}$ that contain both virtual muons and taus, $A_{3}^{(4)}\left(m_{e} / m_{\mu}, m_{e} / m_{\tau}\right)=0$. The mass-independent coefficient has been known for almost fifty years 14:

$A_{1}^{(4)}=-0.32847896557919378 \ldots$

The coefficient of the two-loop mass-dependent contribution to $a_{l}^{\mathrm{QED}}, A_{2}^{(4)}(1 / x)$, with $x=m_{j} / m_{l}$, is generated by the diagram with a vacuum polarization subgraph containing the virtual lepton $j$. This coefficient was first computed in the late 1950 s for the muon $g-2$ with $x=m_{e} / m_{\mu} \ll 1$, neglecting terms of $O(x)$ [15. The exact expression for $0<x<1$ was reported by Elend in 1966 [16]. However, for many years its numerical evaluation was considered tricky because of large cancellations and difficulties in the estimate of the accuracy of the results, so that common practice was to use series expansions instead [17/18/19]. Taking advantage of the properties of the dilogarithm $\mathrm{Li}_{2}(z)=-\int_{0}^{z}(d t / t) \ln (1-t)[20$, the exact result was cast in 21] in a very simple and compact analytic form, valid, contrary to the one in [16], also for $x \geq 1$ (the case relevant to $a_{e}^{\mathrm{QED}}$ and part of $\left.a_{\mu}^{\mathrm{QED}}\right)$. Its numerical evaluation with the mass ratios given in Sec. 2 yields [5]

$A_{2}^{(4)}\left(m_{e} / m_{\mu}\right)=5.19738670(28) \times 10^{-7}$

$A_{2}^{(4)}\left(m_{e} / m_{\tau}\right)=1.83762(60) \times 10^{-9}$,

where the errors are only due to the uncertainties of the mass ratios. The results of Eqs. (5) and (6) are equal to those obtained with a series expansion in powers of the mass ratio $y$ and $\ln y$, with $y \ll 1$ [10].

Adding up Eqs. (4), (5) and (6) one gets the two-loop QED coefficient [5]

$$
\begin{aligned}
C_{e}^{(4)} & =A_{1}^{(4)}+A_{2}^{(4)}\left(m_{e} / m_{\mu}\right)+A_{2}^{(4)}\left(m_{e} / m_{\tau}\right) \\
& =-0.32847844400290(60) .
\end{aligned}
$$

The mass-dependent part of $C_{e}^{(4)}$ is small but not negligible, giving a relative contribution to the theoretical prediction of the electron $g-2$ of 2.4 ppb. This value is much larger than the $0.7 \mathrm{ppb}$ relative uncertainty very recently achieved in the measurement of $a_{e}$ [1]. The uncertainties in $A_{2}^{(4)}\left(m_{e} / m_{\mu}\right)$ and $A_{2}^{(4)}\left(m_{e} / m_{\tau}\right)$ are dominated by those in the latter and were added in quadrature. The resulting error $\delta C_{e}^{(4)}=6 \times 10^{-13}$ leads to a totally negligible $O\left(10^{-18}\right)$ uncertainty in the $a_{e}^{\text {QED }}$ prediction. 
More than one hundred diagrams are involved in the evaluation of the three-loop (sixth-order) QED contribution. Their analytic computation required approximately three decades, ending in the late 1990s. The coefficient $A_{1}^{(6)}$ arises from 72 diagrams. Its exact expression is mainly due to Remiddi and his collaborators 22]23]; its numerical value is

$A_{1}^{(6)}=1.181241456587 \ldots$,

in very good agreement with previous results obtained with numerical methods 24 .

The calculation of the exact expression for the coefficient $A_{2}^{(6)}\left(m_{l} / m_{j}\right)$ for arbitrary values of the mass ratio $m_{l} / m_{j}$ was completed in 1993 by Laporta and Remiddi [25|26] (earlier works include Refs. [27]). Let us focus on $a_{e}^{\mathrm{QED}}(l=e, j=\mu, \tau)$. This coefficient can be further split into two parts: the first one, $A_{2}^{(6)}\left(m_{l} / m_{j}\right.$, vac), receives contributions from 36 diagrams containing either muon or $\tau$ vacuum polarization loops [25], whereas the second one, $A_{2}^{(6)}\left(m_{l} / m_{j}, \mathrm{lbl}\right)$, is due to 12 lightby-light scattering diagrams with either muon or $\tau$ loops [26]. The exact expressions for these coefficients are rather complicated, containing hundreds of polylogarithmic functions up to fifth degree (for the light-by-light diagrams) and complex arguments (for the vacuum polarization ones)they also involve harmonic polylogarithms 28 . Series expansions were provided in Ref. 26 for the cases of physical relevance.

Using the recommended mass ratios given in Sec. 2, the following values were recently computed from the full analytic expressions [5]:

$A_{2}^{(6)}\left(m_{e} / m_{\mu}\right)=-7.37394164(29) \times 10^{-6}$

$A_{2}^{(6)}\left(m_{e} / m_{\tau}\right)=-6.5819(19) \times 10^{-8}$.

Equations (9) and (10) provide the first evaluation of the full analytic expressions for these coefficients with the CODATA mass ratios of 10; they are almost identical to the results $A_{2}^{(6)}\left(m_{e} / m_{\mu}\right)=$ $-7.37394158(28) \times 10^{-6}$ and $A_{2}^{(6)}\left(m_{e} / m_{\tau}\right)=$ $-6.5819(19) \times 10^{-8}$ obtained in [10] via the approximate series expansions in the mass ratios. The small difference between $A_{2}^{(6)}\left(m_{e} / m_{\mu}\right)$ of [10] and Eq. (9) mainly origins from the $O\left(\left(m_{e} / m_{\mu}\right)^{6}\right)$ term in the series expansion of $A_{2}^{(6)}\left(m_{e} / m_{\mu}, \mathrm{lbl}\right)$; indeed, due to its smallness, this term was neglected in the expansions of Ref. [26] used in [10]. The $O\left(\left(m_{e} / m_{\mu}\right)^{6}\right)$ and $O\left(\left(m_{e} / m_{\mu}\right)^{8}\right)$ terms were explicitly provided in Ref. 5 expanding the exact Laporta-Remiddi expression for the sum of light-by-light and vacuum polarization contributions for $m_{l} / m_{j} \ll 1$ (see also [25]29] for parts of these expressions). The value of $A_{2}^{(6)}\left(m_{e} / m_{\mu}\right)$ obtained including these additional terms perfectly agrees with that in Eq. (9) determined with the exact formulae. Indeed, their difference is of $O\left(10^{-23}\right)$, to be compared with the $O\left(10^{-13}\right)$ error $\delta A_{2}^{(6)}\left(m_{e} / m_{\mu}\right)$ due to the present uncertainty of the ratio $m_{e} / m_{\mu}$. Therefore, it will be possible to compute $A_{2}^{(6)}\left(m_{e} / m_{\mu}\right)$ with the simple expansion in Ref. 5]-thus avoiding the complexities of the exact expressions - even if the precision of the ratio $m_{e} / m_{\mu}$ will improve in the future by orders of magnitude.

The contribution of the three-loop diagrams with both $\mu$ and $\tau$ loop insertions in the photon propagator can be calculated numerically from the integral expressions of Ref. 17. The value reported in Ref. 5 is

$A_{3}^{(6)}\left(m_{e} / m_{\mu}, m_{e} / m_{\tau}\right)=1.90945(62) \times 10^{-13}$,

a totally negligible $O\left(10^{-21}\right)$ contribution to $a_{e}^{\mathrm{QED}}$. Adding up Eqs. (8), (9), (10) and (11) one obtains the three-loop QED coefficient [5]

$C_{e}^{(6)}=1.181234016827(19)$.

The relative contribution to $a_{e}^{\mathrm{QED}}$ of the massdependent part of this three-loop coefficient is $\sim 0.1 \mathrm{ppb}$. This is smaller than the present $\sim 0.7$ ppb experimental uncertainty 1 . The error $1.9 \times 10^{-11}$ in Eq. (12) leads to a totally negligible $O\left(10^{-19}\right)$ uncertainty in $a_{e}^{\mathrm{QED}}$.

\subsection{Determination of $\alpha$ from $a_{e}$}

As we already mentioned, recently a new measurement of the electron anomalous magnetic moment by Gabrielse and his collaborators achieved the extremely small relative uncertainty of $0.7 \mathrm{ppb}$, ,

$a_{e}^{\mathrm{EXP}}=1159652180.85(76) \times 10^{-12}$. 
This uncertainty is nearly six times smaller than that of the last measurement of $a_{e}$ reported back in 1987, $a_{e}^{\mathrm{EXP}}=1159652188.3(4.2) \times 10^{-12}$ [30[10]. These two measurements differ by 1.7 standard deviations.

The fine-structure constant $\alpha$ can be determined equating the theoretical SM prediction of the electron $g-2$ with its measured value

$a_{e}^{\mathrm{SM}}(\alpha)=a_{e}^{\mathrm{EXP}}$.

The SM prediction contains the QED contribution $a_{e}^{\mathrm{QED}}(\alpha)=\sum_{i=1}^{5} C_{e}^{(2 i)}(\alpha / \pi)^{i}$ (higher-order coefficients are assumed to be negligible), plus small weak and hadronic loop effects: $a_{e}^{\mathrm{SM}}(\alpha)=$ $a_{e}^{\mathrm{QED}}(\alpha)+a_{e}^{\mathrm{EW}}+a_{e}^{\mathrm{HAD}}$ (the dependence on $\alpha$ of any contribution other than $a_{e}^{\mathrm{QED}}$ is negligible). The EW contribution is 10 :

$a_{e}^{\mathrm{EW}}=0.0297(5) \times 10^{-12} ;$

this precise value includes the two-loop contributions calculated in Refs. 31/32 33. The hadronic term is 103435:

$a_{e}^{\mathrm{HAD}}=1.671(19) \times 10^{-12}$.

The latest value for the four-loop QED coefficient is $C_{e}^{(8)}=-1.7283(35)$ 36]. Following the argument of [10], the educated guess $C_{e}^{(10)}=0.0(3.8)$ for the five-loop coefficient can be adopted. The errors $\delta C_{e}^{(8)}=0.0035$ and $\delta C_{e}^{(10)}=3.8$ lead to an uncertainty of $0.1 \times 10^{-12}$ and $0.3 \times 10^{-12}$ in $a_{e}^{\mathrm{QED}}$, respectively. Solving Eq. (14) with the new measured value of Eq. (13), one obtains 415]

$$
\begin{aligned}
\alpha^{-1} & =137.035999709(12)(30)(2)(90) \\
& =137.035999709(96)[0.70 \mathrm{ppb}] .
\end{aligned}
$$

The first and second errors are due to the uncertainties of the four- and five-loop QED coefficient $\delta C_{e}^{(8)}$ and $\delta C_{e}^{(10)}$, respectively; the third one is caused by the tiny $\delta a_{e}^{\mathrm{HAD}}$, and the last one $(90 \times$ $\left.10^{-9}\right)$ is from the experimental $\delta a_{e}^{\mathrm{EXP}}$ in Eq. (13). The uncertainties of the EW and two/threeloop QED contributions are totally negligible at present.

The amazing precision of the determination in Eq. (17) represents the first significant improvement of this fundamental constant in a decade.
At present, the best determinations of $\alpha$ independent of the electron $g-2$ are

$\alpha^{-1}(\mathrm{Rb})=137.03599878(91)[6.7 \mathrm{ppb}]$,

$\alpha^{-1}(\mathrm{Cs})=137.0360000(11)$ [8.0 ppb];

they are less precise by roughly a factor of ten. The value $\alpha^{-1}(\mathrm{Rb})$ was deduced from the measurement of the ratio $h / M_{\mathrm{Rb}}$ based on Bloch oscillations of $\mathrm{Rb}$ atoms in an optical lattice ( $h$ is the Planck constant and $M_{\mathrm{Rb}}$ is the mass of the $\mathrm{Rb}$ atom) [37, while $\alpha^{-1}(\mathrm{Cs})$ was determined from the measurement of the ratio $h / M_{\mathrm{Cs}}\left(M_{\mathrm{Cs}}\right.$ is the mass of the Cs atom) via cesium recoil measurement techniques 3839. These two determinations of $\alpha$ also rely on the precisely known Rydberg constant and relative atomic masses of the electron, $\mathrm{Rb}$ and $\mathrm{Cs}$ atoms [10]40. The values of $\alpha$ in Eqs. (18) and (19) are in good agreement with the result of Eq. (17), differing from the latter by -1.0 and +0.3 standard deviations, respectively. This comparison provides a beautiful test of the validity of QED. It also probes for possible electron substructure [4].

\section{MUON}

In this section we will summarize the present status of the SM prediction of the muon $g-2$. See Refs. 414221 for recent reviews.

\subsection{QED and Electroweak Contributions}

As we discussed in Secs. 2 3, the one-, two-, and three-loop QED contributions to $a_{l}(l=e$, $\mu$ or $\tau$ ) are known analytically. The four-loop QED part of the muon $g-2$, which is about six times larger than the present experimental uncertainty of $a_{\mu}$, has thus far been evaluated only numerically. This formidable task was first accomplished by Kinoshita and his collaborators in the early 1980s 43 . The latest analyses appeared recently in 36/44. The leading fiveloop QED terms were recently evaluated in [45] and were presented at this workshop [46; estimates obtained with the renormalization-group method agree with these results [47. The total QED prediction currently stands at $a_{\mu}^{\mathrm{QED}}=$ $116584718.09(14)(08) \times 10^{-11}[\underline{5}$. The first error is determined by the uncertainties of the QED co- 
efficients (dominated by the five-loop one), while the second is caused by the tiny uncertainty of the recent value of the fine-structure constant $\alpha$ 4/5].

The EW contribution to $a_{\mu}$ is suppressed by a factor $\left(m_{\mu} / M_{W}\right)^{2}$ with respect to the QED effects. The one-loop part was computed in 1972 by several authors [48]: $a_{\mu}^{\mathrm{EW}}(1$ loop $)=$ $\frac{5 G_{\mu} m_{\mu}^{2}}{24 \sqrt{2} \pi^{2}}\left[1+\frac{1}{5}\left(1-4 \sin ^{2} \theta_{W}\right)^{2}+O\left(m_{\mu}^{2} / M_{Z, W, H}^{2}\right)\right]$, where $G_{\mu}=1.16637(1) \times 10^{-5} \mathrm{GeV}^{-2}$ is the Fermi coupling constant and $\theta_{W}$ is the weak mixing angle. Closed analytic expressions for $a_{\mu}^{\mathrm{EW}}$ (1 loop) taking exactly into account the $m_{\mu}^{2} / M_{B}^{2}$ dependence $(B=Z, W$, Higgs, or other hypothetical bosons) can be found in Refs. 49]. Employing the on-shell definition $\sin ^{2} \theta_{W}=1-M_{W}^{2} / M_{Z}^{2}$ [50, where $M_{Z}=91.1875(21) \mathrm{GeV}$ and $M_{W}$ is the SM prediction of the $W$ mass (which can be derived, for example, from the simple formulae of [51] leading to $M_{W}=80.383 \mathrm{GeV}$ for the Higgs mass $M_{H}=150 \mathrm{GeV}$ ), one obtains $a_{\mu}^{\mathrm{EW}}(1$ loop $)=194.8 \times 10^{-11}$.

The two-loop EW contribution to $a_{\mu}$ is not negligible because of large factors of $\ln \left(M_{Z, W} / m_{f}\right)$, where $m_{f}$ is a fermion mass scale much smaller than $M_{W}$ [52. It was computed in 1995 [3132. The proper treatment of the contribution of the light quarks was addressed in 53154]. These refinements significantly improved the reliability of the fermionic part (that containing closed fermion loops) of $a_{\mu}^{\mathrm{EW}}$ (two-loop) leading, for $M_{H}=$ $150 \mathrm{GeV}$, to $a_{\mu}^{\mathrm{EW}}=154(1)(2) \times 10^{-11}$ [54]. The first error is due to hadronic loop uncertainties, while the second one corresponds to an allowed range of $M_{H} \in[114,250] \mathrm{GeV}$, to the current top mass uncertainty, and to unknown three-loop effects. The leading-logarithm three-loop contribution to $a_{\mu}^{\mathrm{EW}}$ is extremely small [54/55]. The result of [56] for the two-loop bosonic part of $a_{\mu}^{\mathrm{EW}}$, performed without the large $M_{H}$ approximation previously employed in Ref. [31, agrees with this previous evaluation in the large Higgs mass limit.

\subsection{The Hadronic Contribution}

The evaluation of the hadronic leading-order contribution $a_{\mu}^{\mathrm{HLO}}$, due to the hadronic vacuum polarization correction to the one-loop diagram, involves long-distance QCD for which perturbation theory cannot be employed. However, using analyticity and unitarity, it was shown long ago that this term can be computed from hadronic $e^{+} e^{-}$annihilation data via the dispersion integral [57]

$a_{\mu}^{\mathrm{HLO}}=\frac{1}{4 \pi^{3}} \int_{4 m_{\pi}^{2}}^{\infty} d s K_{\mu}(s) \sigma^{(0)}(s)$,

where $\sigma^{(0)}(s)$ is the total cross section for $e^{+} e^{-}$ annihilation into any hadronic state, with extraneous QED corrections subtracted off. The kernel function $K_{\mu}(s)$ decreases monotonically for increasing $s$.

A prominent role among all $e^{+} e^{-}$annihilation measurements is played by the precise data collected in 1994-95 by the CMD-2 detector at the VEPP-2M collider in Novosibirsk for the $e^{+} e^{-} \rightarrow$ $\pi^{+} \pi^{-}$cross section at values of $\sqrt{s}$ between 0.61 and $0.96 \mathrm{GeV}[58$. (quoted systematic error $0.6 \%$, dominated by the uncertainties in the radiative corrections). Recently [59] the CMD-2 Collaboration released its 1996-98 measurements for the same cross section in the full energy range $\sqrt{s} \in[0.37,1.39] \mathrm{GeV}$. The part of these data for $\sqrt{s} \in[0.61,0.96] \mathrm{GeV}$ (quoted systematic error $0.8 \%$ ) agrees with their earlier result published in [58. In 2005, also the SND Collaboration (at the VEPP-2M collider as well) released its analysis of the $e^{+} e^{-} \rightarrow \pi^{+} \pi^{-}$process for $\sqrt{s}$ between 0.39 and $0.98 \mathrm{GeV}$, with a systematic uncertainty of $1.3 \%(3.2 \%)$ for $\sqrt{s}$ larger (smaller) than 0.42 $\mathrm{GeV}$ [60]. However, a recent reanalysis of these data 61] uncovered an error in the treatment of the radiative corrections, reducing the value of the measured cross section. The new SND result appears to be in good agreement with the corresponding one from CMD2.

In 2004 the KLOE experiment at the DAFNE collider in Frascati presented a precise measurement of $\sigma\left(e^{+} e^{-} \rightarrow \pi^{+} \pi^{-}\right)$62 via the initialstate radiation (ISR) method 63] at the $\phi$ resonance. This cross section was extracted for $\sqrt{s}$ between 0.59 and $0.97 \mathrm{GeV}$ with a systematic error of $1.3 \%$ and a negligible statistical one. There are some discrepancies between the KLOE and CMD-2 results, even if their integrated contributions to $a_{\mu}^{\mathrm{HLO}}$ are similar. The data of KLOE [62] 
and SND 61 particularly disagree above the $\rho$ peak, where the latter are significantly higher. The study of the $e^{+} e^{-} \rightarrow \pi^{+} \pi^{-}$process via the ISR method is also in progress at BABAR 64 and Belle 65]. On the theoretical side, analyticity, unitarity and chiral symmetry provide strong constraints for the pion form factor in the low-energy region 66. Perhaps, also lattice QCD computations of $a_{\mu}^{\mathrm{HLO}}$, although not yet competitive with the precise results of the dispersive method, may eventually rival that precision [67].

Recent evaluations of the dispersive integral are in rather good agreement:

$$
\begin{aligned}
& a_{\mu}^{\mathrm{HLO}}=6909(39)_{\exp }(19)_{r a d}(7)_{q c d} \times 10^{-11}[68](21) \\
& a_{\mu}^{\mathrm{HLO}}=6894(42)_{\exp }(18)_{r a d} \times 10^{-11}[69] \\
& a_{\mu}^{\mathrm{HLO}}=6921(56) \times 10^{-11}[70 \\
& a_{\mu}^{\mathrm{HLO}}=6944(48)_{\exp }(10)_{r a d} \times 10^{-11}
\end{aligned}
$$

The three results in Eqs. (21)-(23) already include the recent datasets from CMD-2 [59], SND 61] and BABAR [2] (not all of them, for the value in Eq. (23) ). KLOE's data were not included in the analyses of Refs. 68] and [71]. The preliminary value in Eq. (21) updates the one of Ref. 73. Significant progress is expected from the $e^{+} e^{-}$collider VEPP-2000 under construction in Novosibirsk 74 and, possibly, from DAFNE-2 at Frascati 75 .

The authors of 76 , pioneered the idea of using vector spectral functions derived from the study of hadronic $\tau$ decays $77 / 78$, to improve the evaluation of the dispersive integral. However, the latest analysis with ALEPH, CLEO, and OPAL data yields $a_{\mu}^{\mathrm{HLO}}=7110(50)_{\exp }(8)_{\mathrm{rad}}(28)_{S U(2)} \times$ $10^{-11}$ [73, a value significantly higher than those obtained with $e^{+} e^{-}$data. Isospin-breaking corrections were applied [79. Although the precise CMD-2 $e^{+} e^{-} \rightarrow \pi^{+} \pi^{-}$data are consistent with the corresponding $\tau$ ones for energies below $\sim 0.85 \mathrm{GeV}$, they are significantly lower for larger energies. KLOE's $\pi^{+} \pi^{-}$spectral function confirms this discrepancy with the $\tau$ data. Even if SND's 2005 results 60] were compatible with the $\tau$ ones, their recent reanalysis [61], reducing the value of the measured cross section, seems to indicate that this is no longer the case. Interesting preliminary results from Belle of the study of the decay $\tau^{-} \rightarrow \pi^{-} \pi^{0} \nu_{\tau}$ were presented at this workshop 8081 . This puzzling discrepancy between the $\pi^{+} \pi^{-}$spectral functions from $e^{+} e^{-}$and isospin-breaking-corrected $\tau$ data, also discussed at this workshop 82 , could be caused by inconsistencies in the $e^{+} e^{-}$or $\tau$ data, or in the isospinbreaking corrections which must be applied to the latter 83.

The hadronic higher-order $\left(\alpha^{3}\right)$ contribution $a_{\mu}^{\mathrm{HHO}}$ can be divided into two parts: $a_{\mu}^{\mathrm{HнO}}=$ $a_{\mu}^{\mathrm{HHO}}(\mathrm{vp})+a_{\mu}^{\mathrm{HHO}}(\mathrm{lbl})$. The first one is the $O\left(\alpha^{3}\right)$ contribution of diagrams containing hadronic vacuum polarization insertions 35. Its latest value is $a_{\mu}^{\mathrm{HHO}}(\mathrm{vp})=-97.9(0.9)_{\exp }(0.3)_{\text {rad }} \times 10^{-11}$ [84, 69 ; it changes by $\sim-3 \times 10^{-11}$ if hadronic $\tau$ decay data are used instead 42. The second term, also of $O\left(\alpha^{3}\right)$, is the hadronic light-bylight contribution. As it cannot be directly determined via a dispersion relation approach using data (unlike the hadronic vacuum polarization contribution), its evaluation relies on specific models of low-energy hadronic interactions with electromagnetic currents. Three major components of $a_{\mu}^{\mathrm{HHO}}(\mathrm{lbl})$ can be identified: charged-pion loops, quark loops, and pseudoscalar $\left(\pi^{0}, \eta\right.$, and $\eta^{\prime}$ ) pole diagrams 85. The latter ones dominate the final result and require information on the electromagnetic form factors of the pseudoscalars. In 2001 the authors of [86] uncovered a sign error in earlier evaluations of the dominating pion-pole part. Their estimate of $a_{\mu}^{\mathrm{HHO}}(\mathrm{lbl})$, based also on the previous results for the quark and chargedpions loop parts, is $a_{\mu}^{\mathrm{HHO}}(\mathrm{lbl})=80(40) \times 10^{-11}$. A higher value was obtained in 2003 including shortdistance QCD constraints: $a_{\mu}^{\mathrm{HHO}}(\mathrm{lbl})=136(25) \times$ $10^{-11}$ [87. The upper bounds found in Refs. 88] are consistent with this higher value. Further independent calculations would provide an important check of these results for $a_{\mu}^{\mathrm{HHO}}(\mathrm{lbl})$ (see [89] for a recent critical comparison of these evaluations), a contribution whose uncertainty may become the ultimate limitation of the SM prediction of the muon $g-2$.

\subsection{Standard Model vs. Measurement}

The first column of Table 1 shows $a_{\mu}^{\mathrm{SM}}=$ $a_{\mu}^{\mathrm{QED}}+a_{\mu}^{\mathrm{EW}}+a_{\mu}^{\mathrm{HLO}}+a_{\mu}^{\mathrm{HHO}}$. The values employed for $a_{\mu}^{\mathrm{HLO}}$ are indicated by the reference on the 
Table 1

$a_{\mu}$ : Standard Model vs. measurement.

\begin{tabular}{llll}
\hline \multicolumn{2}{l}{$a_{\mu}^{\mathrm{SM}} \times 10^{11}$} & $\Delta \times 10^{11}$ & $\sigma$ \\
\hline$[68$ & $116591763(60)$ & $317(87)$ & $3.7\langle 3.2\rangle$ \\
69 & $116591748(61)$ & $332(88)$ & $3.8\langle 3.4\rangle$ \\
70 & $116591775(69)$ & $305(93)$ & $3.3\langle 2.8\rangle$ \\
71 & $116591798(63)$ & $282(89)$ & $3.2\langle 2.7\rangle$ \\
\hline 73 & $116591961(70)$ & $119(95)$ & $1.3\langle 0.7\rangle$ \\
\hline
\end{tabular}

left; the value of Ref. 73 was obtained using data from hadronic $\tau$ decays. All $a_{\mu}^{\mathrm{SM}}$ values were derived with $a_{\mu}^{\mathrm{HHO}}(\mathrm{lbl})=80(40) \times 10^{-11} 85[86$. Errors were added in quadrature. The present world average experimental value is $a_{\mu}^{\mathrm{EXP}}=$ $116592080(63) \times 10^{-11}[0.5 \mathrm{ppm}][3]$. Note that the theoretical error on $a_{\mu}$ is now roughly the same as the experimental one. The differences $\Delta=a_{\mu}^{\mathrm{EXP}}-a_{\mu}^{\mathrm{SM}}$ are listed in the second column of Table 1 while the numbers of "standard deviations" $(\sigma)$ appear in the third one. Lower discrepancies, shown in angle brackets, are obtained if $a_{\mu}^{\mathrm{HHO}}(\mathrm{lbl})=136(25) \times 10^{-11}$ [87] is used instead of $80(40) \times 10^{-11}$ 85186.

\section{TAU}

This section summarizes the results reported at this workshop [90 for the theoretical prediction of the $\tau$ lepton $g-2$. We also refer the reader to Ref. 91] for a very recent update and review.

\subsection{QED and Electroweak Contributions}

The sum of the one-, two- and three-loop QED contribution to $a_{\tau}$, obtained using the mass ratios given in Sec. 2 is [5]:

$a_{\tau}^{\mathrm{QED}}=117324(2) \times 10^{-8}$.

The error $\delta a_{\tau}^{\mathrm{QED}}$ is the uncertainty $\delta C_{\tau}^{(8)}(\alpha / \pi)^{4} \sim$ $\pi^{2} \ln ^{2}\left(m_{\tau} / m_{e}\right)(\alpha / \pi)^{4} \sim 2 \times 10^{-8}$ assigned in [5] to $a_{\tau}^{\mathrm{QED}}$ for uncalculated four-loop contributions. The errors due to the uncertainties of the $O\left(\alpha^{2}\right)\left(5 \times 10^{-10}\right)$ and $O\left(\alpha^{3}\right)$ terms $\left(3 \times 10^{-11}\right)$, as well as that induced by the uncertainty of $\alpha$ $\left(8 \times 10^{-13}\right)$ are negligible. The result in Eq. (25) supersedes the earlier values $a_{\tau}^{\mathrm{QED}}=117319(1) \times$
$10^{-8} 92$ and $a_{\tau}^{\mathrm{QED}}=117327.1(1.2) \times 10^{-8} \underline{93}$

(see Refs. 5191] for details).

The EW correction to the anomalous magnetic moment of the $\tau$ lepton is of the same order of magnitude as the three-loop QED one. The oneloop EW term is $a_{\tau}^{\mathrm{EW}}(1 \mathrm{loop})=55.1 \times 10^{-8}$ [8]. The estimate of the total EW contribution of Ref. 92] $a_{\tau}^{\mathrm{EW}}=55.60(2) \times 10^{-8}$, obtained from the one-loop formula, is similar to the one-loop value reported above. However, it doesn't contain the two-loop contribution, which is not negligible.

As we already discussed for the muon $g-2$, the two-loop EW contributions $a_{l}^{\mathrm{EW}}$ (2 loop) $(l=e, \mu$ or $\tau$ ) were computed in 1995 [31/32 33, leading to a significant reduction of the one-loop prediction because of large factors of $\ln \left(M_{Z, W} / m_{f}\right)$, where $m_{f}$ is a fermion mass scale much smaller than $M_{W}$ [52. The numerical value of the bosonic part of $a_{\tau}^{\mathrm{EW}}\left(2\right.$ loop), for $M_{H}=150 \mathrm{GeV}$, is $a_{\tau}^{\mathrm{EW}}(2$ loop bos $)=-3.06 \times 10^{-8}$ [3133 90,91]. Very recently, the analysis of the fermionic part of Ref. 32 33 was slightly refined in Ref. 91] leading, for $M_{H}=150 \mathrm{GeV}$, to $a_{\tau}^{\mathrm{EW}}(2$ loop fer $)=$ $-4.68 \times 10^{-8}$. The sum of the fermionic and bosonic two-loop EW contributions gives $a_{\tau}^{\mathrm{EW}}(2$ loop $)=-7.74 \times 10^{-8}$ 9091, a $14 \%$ reduction of the one-loop result. As discussed in Sec. 4 the leading-logarithm three-loop EW contributions to the muon $g-2$ were determined to be extremely small via renormalization-group analyses [54/55]; in Ref. [9091 an additional uncertainty of $O\left[a_{\tau}^{\mathrm{EW}}(2\right.$ loop $\left.)(\alpha / \pi) \ln \left(M_{z}^{2} / m_{\tau}^{2}\right)\right] \sim$ $O\left(10^{-9}\right)$ was assigned to $a_{\tau}^{\mathrm{EW}}$ to account for these neglected three-loop effects. Adding $a_{\tau}^{\mathrm{EW}}(2$ loop) to the one-loop value presented above, one gets the total EW correction $\left(M_{H}=150 \mathrm{GeV}\right)$ 9091]:

$a_{\tau}^{\mathrm{EW}}=47.4(5) \times 10^{-8}$.

The uncertainty allows $M_{H}$ to range from 114 $\mathrm{GeV}$ up to $\sim 300 \mathrm{GeV}$, and reflects the estimated errors induced by hadronic loop effects, neglected two-loop bosonic terms and the missing threeloop contribution. It also includes the tiny errors due to the uncertainties in $M_{\text {top }}$ and $m_{\tau}$. The value in Eq. (26) is in agreement with the prediction $a_{\tau}^{\mathrm{EW}}=47(1) \times 10^{-8} 3393$, with a reduced uncertainty. As we mentioned earlier, the EW estimate of Ref. [92], $a_{\tau}^{\mathrm{EW}}=55.60(2) \times 10^{-8}$, mainly 
differs from Eq. (26) in that it doesn't include the two-loop corrections.

\subsection{The Hadronic Contribution}

Similarly to the case of the muon, the leadingorder hadronic contribution to the $\tau$ lepton $g-2$ is given by the dispersion integral in Eq. (20) (with the kernel $K_{\mu}(s)$ replaced by $K_{\tau}(s)$ ), in which the role of the low energies is very important, although not as strongly as in $a_{\mu}^{\mathrm{HLO}}$. The history of the $a_{\tau}^{\mathrm{HLO}}$ calculations 91929394959697] based mainly on experimental $e^{+} e^{-}$data is shown in Table 2. Purely theoretical estimates somewhat undervalue the hadronic contribution and have rather large uncertainties 9899100101.

Table 2

Calculations of $\mathrm{a}_{\tau}^{\mathrm{HLO}}$.

\begin{tabular}{lc}
\hline Author & $\mathrm{a}_{\tau}^{\mathrm{HLO}} \times 10^{8}$ \\
\hline Narison [94] & $370 \pm 40$ \\
Barish \& Stroynowski 99] & $\sim 350$ \\
Samuel et al. [92] & $360 \pm 32$ \\
Eidelman \& Jegerlehner [96]97] & $338.4 \pm 2.0 \pm 9.1$ \\
Narison [93] & $353.6 \pm 4.0$ \\
Eidelman \& Passera 9091] & $337.5 \pm 3.7$ \\
\hline
\end{tabular}

The calculation of the leading-order contribution was very recently updated using the whole bulk of experimental data below $12 \mathrm{GeV}$, which include, as discussed in Sec. 4, old data compiled in Refs. 9673, and recent results from CMD2 [59], SND 61], KLOE 62] and BABAR 72]. The improvement is particularly strong in the channel $e^{+} e^{-} \rightarrow \pi^{+} \pi^{-}$. The result of this analysis is [9091]:

$a_{\tau}^{\mathrm{HLO}}=337.5(3.7) \times 10^{-8}$.

The overall uncertainty is 2.5 times smaller than that of the previous data-based prediction 9697.

Like $a_{\mu}^{\mathrm{HHO}}$, the hadronic higher-order $\left(\alpha^{3}\right)$ contribution $a_{\tau}^{\mathrm{HHO}}$ can be divided into two parts: $a_{\tau}^{\mathrm{HHO}}=a_{\tau}^{\mathrm{HHO}}(\mathrm{vp})+a_{\tau}^{\mathrm{HHO}}(\mathrm{lbl})$. The first one, the $O\left(\alpha^{3}\right)$ contribution of diagrams containing hadronic self-energy insertions in the photon propagators, is 35]:

$a_{\tau}^{\mathrm{HHO}}(\mathrm{vp})=7.6(2) \times 10^{-8}$.
Note that naïvely rescaling the muon result by the factor $m_{\tau}^{2} / m_{\mu}^{2}$ leads to the totally incorrect estimate $a_{\tau}^{\text {нно }}(\mathrm{vp})=\left(-101 \times 10^{-11}\right) \times m_{\tau}^{2} / m_{\mu}^{2}=$ $-29 \times 10^{-8}$ (the $a_{\mu}^{\mathrm{HHO}}(\mathrm{vp})$ value is from Ref. [35]); even the sign is wrong! Until recently, very few estimates of the light-by-light contribution $a_{\tau}^{\mathrm{HHO}}(\mathrm{lbl})$ existed in the literature 92 9335, and all of them were obtained simply rescaling the muon results $a_{\mu}^{\mathrm{HHO}}(\mathrm{lbl})$ by a factor $m_{\tau}^{2} / m_{\mu}^{2}$. These very naïve estimates fall short of what is needed, as this scaling is not justified. For these reasons, a parton-level estimate of $a_{\tau}^{\mathrm{HHO}}(\mathrm{lbl})$ was recently performed in Ref. [91, obtaining

$a_{\tau}^{\mathrm{HHO}}(\mathrm{lbl})=5(3) \times 10^{-8}$.

This value is much lower than those obtained by simple rescaling. We refer the reader to Ref. 91] for a detailed discussion.

\subsection{Standard Model prediction for $\boldsymbol{a}_{\boldsymbol{\tau}}$}

We can now add up all the contributions discussed in the previous sections to derive the SM prediction for $a_{\tau}$ 9091:

$$
\begin{aligned}
a_{\tau}^{\mathrm{SM}} & =a_{\tau}^{\mathrm{QED}}+a_{\tau}^{\mathrm{EW}}+a_{\tau}^{\mathrm{HLO}}+a_{\tau}^{\mathrm{HHO}} \\
& =117721(5) \times 10^{-8} .
\end{aligned}
$$

Errors were added in quadrature. Comparing the most stringent experimental limit mentioned above, $-0.052<a_{\tau}^{\mathrm{EXP}}<0.013$ at $95 \%$ confidence level [7, with Eq. (30), it is clear that the sensitivity of the best existing measurements is still more than an order of magnitude worse than needed. For other limits on $a_{\tau}$ see Ref. [13102.

\section{CONCLUSIONS AND OUTLOOK}

The study of the anomalous magnetic moment of the electron and the muon provides a beautiful example of interplay between theory and experiment. It started more than 50 years ago: Schwinger's 1948 calculation 9] of the leading contribution to $a_{e}$ was one of the very first results of QED, and its agreement with the experimental value provided one of the early confirmations of this theory. In July 2006 Gabrielse and his collaborators published their measurement of $a_{e}$ with an unprecedented accuracy of $0.7 \mathrm{ppb}$ [1, breaking the 1987 world record held by Dehmelt and his 
collaborators 30. On the theoretical side, heroic higher-order QED calculations were performed over the years by Kinoshita, Remiddi, and their collaborators. A result of this extraordinary synergy is today's determination of the fine-structure constant via $a_{e}, \alpha^{-1}=137.035999709$ (96) 415, whose uncertainty is roughly ten times smaller than that from any other method. A further reduction of the experimental error $\delta a_{e}^{\mathrm{EXP}}$, which dominates the uncertainty of $\alpha$, appears to be at hand 114.

The SM prediction of the muon $g-2$ deviates from the present experimental value by more than $3 \sigma$, if data from $e^{+} e^{-}$collisions are employed to evaluate the leading-order hadronic term (the recent datasets from the CMD-2 59, SND [61] and BABAR [72] are already included). This deviation cannot be ignored. Determinations based on $\tau$-decay data deviate only by approximately $1 \sigma$. The puzzling discrepancy between the $\pi^{+} \pi^{-}$spectral functions from $e^{+} e^{-}$ and isospin-breaking-corrected $\tau$ data could be caused by inconsistencies in the $e^{+} e^{-}$or $\tau$ data, or in the isospin-breaking corrections applied to the latter. Indeed, some disagreements occur between $e^{+} e^{-}$data sets, requiring further detailed investigations. On the other hand, the connection of $\tau$ data with the leading hadronic contribution to $a_{\mu}$ is less direct, and one wonders whether all possible isospin-breaking effects have been properly taken into account.

The impressive results of the E821 experiment are still limited by statistical errors. A new experiment, E969, has been approved (but not yet funded) at Brookhaven in 2004 103. Its goal is to reduce the present experimental uncertainty by a factor of 2.5 to about $0.2 \mathrm{ppm}$. A letter of intent for an even more precise muon $g-2$ experiment was submitted to J-PARC with the proposal to reach a precision below $0.1 \mathrm{ppm}$ 104. While the QED and EW contributions appear to be ready to rival these precisions, much effort will be needed to reduce the hadronic uncertainty by a factor of two. This effort is challenging but possible, and certainly well motivated by the excellent opportunity the muon $g-2$ is providing us to unveil (or constrain) NP effects.

The $g-2$ of the $\tau$ lepton is even more sensitive than the muon one to EW and NP loop effects that give contributions $\sim m_{l}^{2}$. However, unfortunately, the very short lifetime of the $\tau$ lepton makes it very difficult to determine $a_{\tau}$ by measuring its spin precession in the magnetic field, like in the muon $g-2$ experiment [2]3. Instead, experiments focus on high-precision measurements of the $\tau$ lepton pair production in various highenergy processes and comparison of the measured cross sections with the QED predictions [7]13, but their sensitivity is still more than an order of magnitude worse than that required to determine $a_{\tau}$.

Nonetheless, there are many interesting suggestions to measure $a_{\tau}$, e.g., from the radiation amplitude zero in radiative $\tau$ decays [105] or from other observables; these methods could possibly exploit the very large $\tau$ lepton samples collected at $B$ factories. Reference [106 suggests a similar method to study $a_{\tau}$ using radiative $W$ decays and potentially very high data samples at LHC. Yet another method would use the channeling in a bent crystal similarly to the measurement of magnetic moments of short-living baryons 107 . In the case of the $\tau$ lepton, it was suggested to use the decay $B^{+} \rightarrow \tau^{+} \nu_{\tau}$, which would produce polarized $\tau$ leptons 92 and was recently observed 108 . We believe that a detailed feasibility study of such experiments, as well as further attempts to improve the accuracy of the theoretical prediction for $a_{\tau}$, are quite timely.

\section{ACKNOWLEDGMENTS}

I would like to thank the organizers for their excellent coordination of this workshop, and in particular A. Lusiani and S. Eidelman for their kind invitation. I am also grateful to S. Eidelman, M. Giacomini and F.V. Ignatov for many fruitful discussions and collaborations.

\section{REFERENCES}

1. B. Odom, D. Hanneke, B. D'Urso, and G. Gabrielse, Phys. Rev. Lett. 97 (2006) 030801.

2. H.N. Brown et al., Phys. Rev. D 62 (2000) 91101; Phys. Rev. Lett. 86 (2001) 2227; 
G.W. Bennett et al., ibid. 89 (2002) 101804; ibid. 89 (2002) 129903 (E).

3. G.W. Bennett et al., Phys. Rev. Lett. 92 (2004) 161802; Phys. Rev. D 73 (2006) 072003 .

4. G. Gabrielse, D. Hanneke, T. Kinoshita, M. Nio, and B. Odom, Phys. Rev. Lett. 97 (2006) 030802.

5. M. Passera, Phys. Rev. D 75 (2007) 013002.

6. A. Czarnecki and W.J. Marciano, Phys. Rev. D 64 (2001) 013014.

7. J. Abdallah et al., Eur. Phys. J. C35 (2004) 159 .

8. T. Kinoshita, W.J. Marciano, in Quantum Electrodynamics, edited by T. Kinoshita (World Scientific, 1990), pp. 419-478.

9. J.S. Schwinger, Phys. Rev. 73 (1948) 416.

10. P.J. Mohr and B.N. Taylor, Rev. Mod. Phys. 77 (2005) 1.

11. K. Hagiwara et al. [Particle Data Group], Phys. Rev. D 66 (2002) 010001.

12. S. Eidelman et al. [Particle Data Group], Phys. Lett. B 592 (2004) 1.

13. W.-M. Yao et al. [Particle Data Group], J. Phys. G 33 (2006) 1.

14. C.M. Sommerfield, Phys. Rev. 107 (1957) 328; Ann. Phys. (N.Y.) 5 (1958) 26; A. Petermann, Helv. Phys. Acta 30 (1957) 407; Nucl. Phys. 5 (1958) 677.

15. H. Suura and E. Wichmann, Phys. Rev. 105 (1957) 1930; A. Petermann, Phys. Rev. 105 (1957) 1931.

16. H.H. Elend, Phys. Lett. 20 (1966) 682; 21 (1966) 720(E).

17. M.A. Samuel and G. Li, Phys. Rev. D 44 (1991) 3935; 46 (1992) 4782(E); 48 (1993) 1879(E).

18. G. Li, R. Mendel and M.A. Samuel, Phys. Rev. D 47 (1993) 1723.

19. A. Czarnecki, M. Skrzypek, Phys. Lett. B449 (1999) 354.

20. L. Lewin, Polylogarithms and Associated Functions, Elsevier North Holland, 1981.

21. M. Passera, J. Phys. G 31 (2005) R75.

22. J.A. Mignaco, E. Remiddi, Nuovo Cim. A 60 (1969) 519; R. Barbieri, E. Remiddi, Phys. Lett. B 49 (1974) 468; R. Barbieri, M. Caffo, E. Remiddi, ibid. 57 (1975)
460; M.J. Levine, E. Remiddi, R. Roskies, Phys. Rev. D 20 (1979) 2068; S. Laporta, E. Remiddi, Phys. Lett. B 265 (1991) 182; S. Laporta, Phys. Rev. D 47 (1993) 4793; Phys. Lett. B 343 (1995) 421; S. Laporta, E. Remiddi, Phys. Lett. B 356 (1995) 390.

23. S. Laporta and E. Remiddi, Phys. Lett. B 379 (1996) 283.

24. T. Kinoshita, Phys. Rev. Lett. 75 (1995) 4728; Quantum Electrodynamics, edited by T. Kinoshita (World Scientific, Singapore, 1990), pp. 218-321.

25. S. Laporta, Nuovo Cim. A 106 (1993) 675.

26. S. Laporta and E. Remiddi, Phys. Lett. B 301 (1993) 440.

27. T. Kinoshita, Nuovo Cim. B 51 (1967) 140; B.E. Lautrup, E. de Rafael, Phys. Rev. 174 (1968) 1835; R. Barbieri, E. Remiddi, Nucl. Phys. B 90 (1975) 233; B.E. Lautrup, M.A. Samuel, Phys. Lett. B 72 (1977) 114.

28. E. Remiddi and J.A.M. Vermaseren, Int. J. Mod. Phys. A 15 (2000) 725; T. Gehrmann and E. Remiddi, Comput. Phys. Commun. 141 (2001) 296; 144 (2002) 200; D. Maître, Comput. Phys. Commun. 174 (2006) 222.

29. J.H. Kühn et al., Phys. Rev. D 68 (2003) 033018.

30. R.S. Van Dyck, P.B. Schwinberg and H.G. Dehmelt, Phys. Rev. Lett. 59 (1987) 26.

31. A. Czarnecki, B. Krause and W.J. Marciano, Phys. Rev. Lett. 76 (1996) 3267.

32. A. Czarnecki, B. Krause and W.J. Marciano, Phys. Rev. D 52 (1995) 2619.

33. A. Czarnecki and B. Krause, Nucl. Instrum. Meth. A 389 (1997) 314.

34. M. Davier and A. Höcker, Phys. Lett. B 435 (1998) 427.

35. B. Krause, Phys. Lett. B 390 (1997) 392.

36. T. Kinoshita and M. Nio, Phys. Rev. D 73 (2006) 013003.

37. P. Cladé et al., Phys. Rev. Lett. 96 (2006) 033001.

38. A. Wicht et al., in Proc. of the 6th Symp. on Freq. Standards and Metrology (World Scientific, Singapore, 2002), pp. 193-212; Phys. Scripta T 102 (2002) 82.

39. V. Gerginov et al., Phys. Rev. A 73 (2006) 032504 . 
40. M.P. Bradley et al., Phys. Rev. Lett. 83 (1999) 4510.

41. K. Melnikov and A. Vainshtein, Theory of the muon anomalous magnetic moment, Springer, 2006; M. Passera, Nucl. Phys. Proc. Suppl. 155 (2006) 365; M. Knecht, hep-ph/0307239 A. Nyffeler, Acta Phys. Polon. B 34 (2003) 5197; K. Melnikov, Int. J. Mod. Phys. A 16 (2001) 4591; V.W. Hughes and T. Kinoshita, Rev. Mod. Phys. 71 (1999) S133; A. Czarnecki and W.J. Marciano, Nucl. Phys. Proc. Suppl. 76 (1999) 245.

42. M. Davier and W.J. Marciano, Annu. Rev. Nucl. Part. Sci. 54 (2004) 115;

43. T. Kinoshita, W.B. Lindquist, Phys. Rev. Lett. 47 (1981) 1573; T. Kinoshita, B. Nizic, Y. Okamoto, Phys. Rev. Lett. 52 (1984) 717.

44. T. Kinoshita and M. Nio, Phys. Rev. D 70 (2004) 113001.

45. T. Kinoshita and M. Nio, Phys. Rev. D 73 (2006) 053007.

46. M. Nio, these proceedings.

47. A.L. Kataev, Phys. Lett. B 284 (1992) 401; Nucl. Phys. Proc. Suppl. 155 (2006) 369; hep-ph/0602098 Phys. Rev. D 74 (2006) 073011; A.L. Kataev and V.V. Starshenko, Phys. Rev. D 52 (1995) 402.

48. R. Jackiw and S. Weinberg, Phys. Rev. D 5 (1972) 2396; I. Bars and M. Yoshimura, Phys. Rev. D6 (1972) 374; G. Altarelli, N. Cabibbo and L. Maiani, Phys. Lett. B40 (1972) 415; W.A. Bardeen, R. Gastmans and B. Lautrup, Nucl. Phys. B46 (1972) 319; K. Fujikawa, B.W. Lee and A.I. Sanda, Phys. Rev. D 6 (1972) 2923.

49. A.I. Studenikin, Fiz. Elem. Chast. Atom. Yadra 21 (1990) 605; hep-ph/9808219; Yad. Fiz. 62 (1999) 2248.

50. A. Sirlin, Phys. Rev. D 22 (1980) 971.

51. G. Degrassi et al., Phys. Lett. B 418 (1998) 209; G. Degrassi and P. Gambino, Nucl. Phys. B 567 (2000) 3; A. Ferroglia et al., Phys. Rev. D 65 (2002) 113002; M. Awramik et al., Phys. Rev. D 69 (2004) 053006; Phys. Rev. Lett. 93 (2004) 201805.

52. T.V. Kukhto et al., Nucl. Phys. B 371 (1992) 567.
53. S. Peris, M. Perrottet and E. de Rafael, Phys. Lett. B 355 (1995) 523; M. Knecht, S. Peris, M. Perrottet and E. de Rafael, JHEP 0211 (2002) 003.

54. A. Czarnecki, W.J. Marciano and A. Vainshtein, Phys. Rev. D 67 (2003) 073006.

55. G. Degrassi, G.F. Giudice, Phys. Rev. D 58 (1998) 053007.

56. S. Heinemeyer, D. Stöckinger and G. Weiglein, Nucl. Phys. B699 (2004) 103; T. Gribouk and A. Czarnecki, Phys. Rev. D 72 (2005) 53016.

57. C. Bouchiat and L. Michel, J. Phys. Radium 22 (1961) 121; M. Gourdin and E. de Rafael, Nucl. Phys. B 10 (1969) 667.

58. R.R. Akhmetshin et al., Phys. Lett. B 578 (2004) 285.

59. V.M. Aulchenko et al., JETP Lett. 82 (2005) 743; V.M. Aulchenko et al., JETP Lett. 84 (2006) 413; R.R. Akhmetshin et al., hep-ex/0610021; S. Eidelman, these proceedings.

60. M.N. Achasov et al., J. Exp. Theor. Phys. 101 (2005) 1053.

61. M.N. Achasov et al., J. Exp. Theor. Phys. 103 (2006) 380.

62. A. Aloisio et al., Phys. Lett. B 606 (2005) 12; G. Venanzoni, these proceedings.

63. G. Rodrigo, these proceedings; W. Wang, these proceedings.

64. V. Druzhinin, hep-ex/0601020

65. B.A. Shwartz, Nucl. Phys. Proc. Suppl. 144 (2005) 245.

66. G. Colangelo, Nucl. Phys. Proc. Suppl. 131 (2004) 185.

67. T. Blum, Phys. Rev. Lett. 91 (2003) 052001; M. Gockeler et al., Nucl. Phys. B 688 (2004) 135; C. Aubin and T. Blum, PoS LAT2005 (2006) 089; hep-lat/0608011.

68. S. Eidelman, proceedings of the XXXIII International Conference on High Energy Physics, July 27-August 2 2006, Moscow (Russia), World Scientific, to appear; M. Davier, these proceedings.

69. K. Hagiwara, A.D. Martin, D. Nomura and T. Teubner, hep-ph/0611102.

70. F. Jegerlehner, Nucl. Phys. Proc. Suppl. 162 (2006) 22. 
71. J.F. de Trocóniz and F.J. Ynduráin, Phys. Rev. D 71 (2005) 073008.

72. B. Aubert et al., Phys. Rev. D 69 (2004) 011103; ibid. D 70 (2004) 072004; ibid. D 71 (2005) 052001; ibid. D 73 (2006) 052003.

73. M. Davier, S. Eidelman, A. Höcker and Z. Zhang, Eur. Phys. J. C 31 (2003) 503.

74. I.A. Koop et al., Frascati Physics Series Vol. XVI (1999) 393; AIP Conf. Proc. 588 (2001) 319; S. Eidelman, these proceedings.

75. F. Ambrosino et al., hep-ex/0603056

76. R. Alemany, M. Davier and A. Höcker, Eur. Phys. J. C 2 (1998) 123.

77. M. Davier, A. Höcker and Z. Zhang, Rev. Mod. Phys. 78 (2006) 1043.

78. J. Portolés, these proceedings.

79. W.J. Marciano and A. Sirlin, Phys. Rev. Lett. 61 (1988) 1815; V. Cirigliano, G. Ecker and H. Neufeld, Phys. Lett. B 513 (2001) 361; JHEP 0208 (2002) 002.

80. K. Abe et al., hep-ex/0512071; H. Hayashii, PoS HEP2005 (2006) 291.

81. M. Fujikawa, these proceedings.

82. K. Maltman, these proceedings; F. FloresTlalpa, F. Flores-Baez, G.L. Castro and G.T. Sanchez, these proceedings, hep-ph/0611226.

83. S. Ghozzi and F. Jegerlehner, Phys. Lett. B 583, 222 (2004); M. Davier, Nucl. Phys. Proc. Suppl. 131 (2004) 123; K. Maltman, Phys. Lett. B 633 (2006) 512; K. Maltman and C. E. Wolfe, Phys. Rev. D 73 (2006) 013004; F. Flores-Baez, A. Flores-Tlalpa, G. Lopez Castro and G. Toledo Sanchez, Phys. Rev. D 74 (2006) 071301.

84. K. Hagiwara, A.D. Martin, D. Nomura and T. Teubner, Phys. Rev. D 69 (2004) 093003.

85. J. Bijnens, E. Pallante and J. Prades, Nucl. Phys. B 474 (1996) 379; ibid. B 626 (2002) 410; M. Hayakawa and T. Kinoshita, Phys. Rev. D 57 (1998) 465; ibid. D 66 (2002) 019902(E).

86. M. Knecht and A. Nyffeler, Phys. Rev. D 65 (2002) 73034; M. Knecht, A. Nyffeler, M. Perrottet and E. de Rafael, Phys. Rev. Lett. 88 (2002) 71802; A. Nyffeler, Acta Phys. Polon. B 34 (2003) 5197.

87. K. Melnikov and A. Vainshtein, Phys. Rev.
D 70 (2004) 113006; A. Vainshtein, these proceedings.

88. J. Erler and G.T. Sanchez, Phys. Rev. Lett. 97 (2006) 161801; A.A. Pivovarov, Phys. At. Nucl. 66 (2003) 902 [Yad. Fiz. 66 (2003) 934].

89. J. Bijnens and J. Prades, hep-ph/0701240.

90. S. Eidelman, M. Giacomini, F. Ignatov and M. Passera, these proceedings.

91. S. Eidelman and M. Passera, Mod. Phys. Lett. A 22 (2007) 159.

92. M.A. Samuel, G. Li and R. Mendel, Phys. Rev. Lett. 67 (1991) 668; 69 (1992) 995(E).

93. S. Narison, Phys. Lett. B 513 (2001) 53; 526 (2002) 414(E).

94. S. Narison, J. Phys. G 4 (1978) 1849.

95. B.C. Barish and R. Stroynowski, Phys. Rep. 157 (1988) 1.

96. S. Eidelman and F. Jegerlehner, Z. Phys. C 67 (1995) 585.

97. F. Jegerlehner, Nucl. Phys. Proc. Suppl. 51 (1996) 131.

98. M. Benmerrouche, G. Orlandini, T.G. Steele, Phys. Lett. B 316 (1993) 381.

99. F. Hamzeh and N.F. Nasrallah, Phys. Lett. B 373 (1993) 211.

100. B. Holdom, R. Lewis and R.R. Mendel, Z. Phys. C 63 (1994) 71.

101. A.E. Dorokhov, Acta Phys. Polon. B 36 (2005) 3751.

102. G.A. Gonzalez-Sprinberg, A. Santamaria and J. Vidal, Nucl. Phys. B 582 (2000) 3.

103. R.M. Carey et al., Proposal of the BNL Experiment E969, 2004; B.L. Roberts, hep-ex/0501012 Nucl. Phys. Proc. Suppl. 155 (2006) 372; D.W. Hertzog, these proceedings, hep-ex/0611025.

104. J-PARC L.o.I. L17, B.L. Roberts contact person.

105. M.L. Laursen, M.A. Samuel and A. Sen, Phys. Rev. D 29 (1984) 2652; ibid. D 56 (1997) 3155(E).

106. M.A. Samuel and G. Li, Int. J. Theor. Phys. 33 (1994) 1471.

107. D. Chen et al., Phys. Rev. Lett. 68 (1992) 3286.

108. K. Ikado et al., Phys. Rev. Lett. 97 (2006) 251802 . 